\title{
Research on Cloud Computing Network Architecture Based on SDN Technology
}

\author{
Weibo Li \\ wuhan textile university, Wuhan, 430073, China \\ leewb@wtu.edu.cn
}

Keywords: Cloud computing; Software defined networking; Network virtualization; System
architecture

\begin{abstract}
Traditional internet data center is shifted to cloud computing data center with rapid development of mobile Internet and cloud computing currently. Cloud computing network burden is aggravated as a result. More optimal cloud computing network architecture is required for replacing traditional cloud computing network architecture aiming at current technical difficulties such as network base flow control, address reuse, access control, security control, automatic network configuration, etc. Software defined networking (SDN) technology, as a new service level network technology, is one of feasible and practical methods to solve current cloud computing network problems. In the paper, cloud computing technology and main problems thereof are firstly analyzed. Thoughts for solving the problems are proposed. After SDN technical plans are analyzed, cloud computing architecture based on Overlay SDN technology is proposed; and development trend of cloud computing network is discussed finally.
\end{abstract}

\section{Introduction}

How to improve computing and data storage ability has become a problem that should be solved by network computing in the future urgently with the advent of big data era. Cloud computing, as a network computing technology, gradually integrates data parallel processing, grid computing, distributed computing and other technologies in recent years, it can achieve request-response access to shared resource pool and other function anytime and anywhere. Users only need to send request, and cloud service provider can provide users with computing resources, storage space, other application software, etc. through cloud computing, and user terminal equipment can become simpler and more practical [1].

Cloud computing technology has the following main advantages: high economic and practical performance, high extension, high reliability, high convenience and supply of on-demand service. However, wider and wider application of cloud computing technology, many problems in traditional network are more and more prominent. In traditional cloud computing network, users need to configure different network level structure such as switches, subnet, Access Control Lists (ACLs) [2], etc. Therefore, cloud application can be normally used. In the paper, it is proposed that Overlay SDN technology is utilized as a part of cloud computing. Complicated configuration problem in traditional cloud computing networks can be solved. Highly-automatic connection performance can be actually realized. Software Defined Network (SDN) is adopted for separating cloud network control layer and data layer. Programmable interface can be provided for users in network control layer. Users can realize dynamic monitoring and management on cloud network through interface by the aid of programming mode according to own demand on function [3]. Meanwhile, uses can realize flexible configuration of network resources, and the main platform architecture is shown in figure 1. 


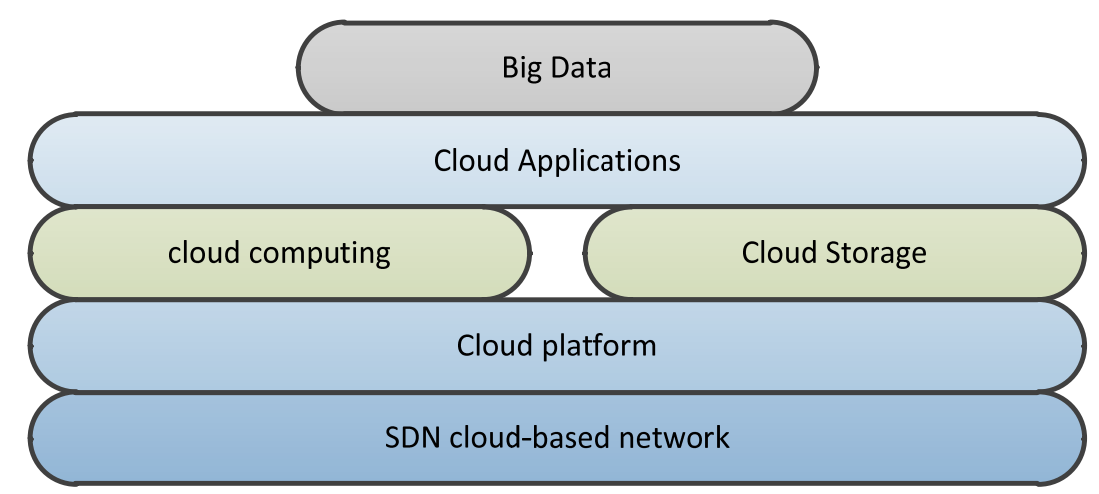

Fig.1. Cloud platform architecture based on SDN cloud computing network

\section{Cloud computing network architecture}

Cloud computing network aims at improving data throughput of the network system through utilizing distributed resources, thereby reaching the purpose of processing large-scale and complicated calculations[4]. Cloud computing network architecture belongs to a novel network architecture which is established on the programmable basis, and realizes resource integration and distribution according to user demand through using the unified platform for operation and management. In the whole cloud computing system, the system is usually divided into two parts of front end and rear end. System front end refers to the part which can be directly observed by users. System rear end is regarded ad cloud system, and the two parts are mutually connected through network, which supplement each other. Information can be connected to the cloud end in the front end of the cloud computing system through user terminal equipment and application request, and business of cloud computing network is processed in the rear end through utilizing large-scale server, data storage and other equipment. Traditional cloud computing data center network design is shown in figure 2.

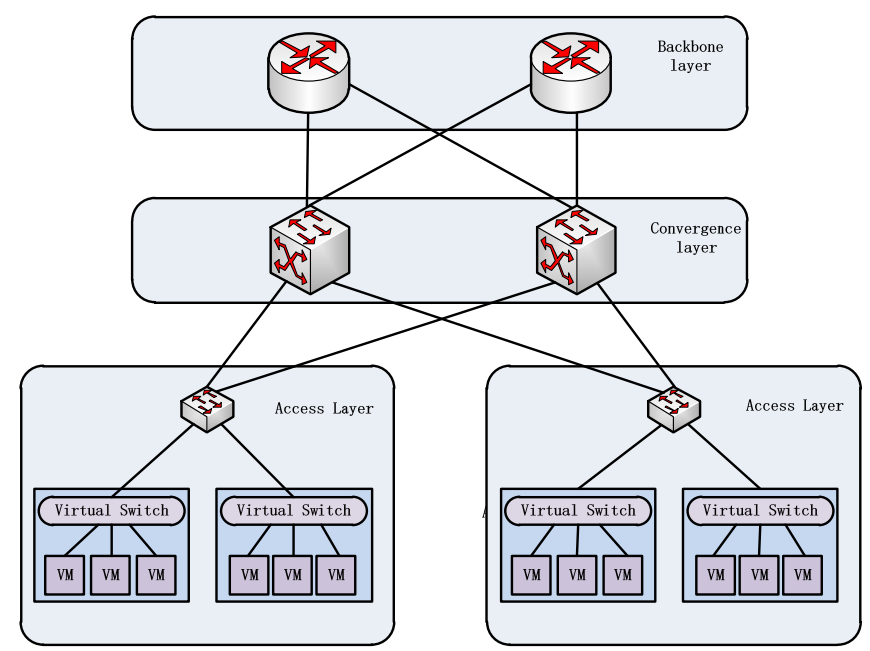

Fig.2. Network architecture of traditional three-layer cloud computing data center

Cloud computing data center network respectively consists of access layer, convergence layer and backbone layer, wherein the virtual switch is responsible for data switch of server internal virtual machine and treatment of data fed in and discharged out of the server. Data traffic is introduced into cloud computing network through access layer. There are three-layer routings among access layer, convergence layer and backbone layer, and forwarding function to data can be ensured through protocol [5].

The traditional three-layer cloud computing data center network architecture has many problems in current cloud computing platform. It is difficult to adapt to current development of cloud computing technology. Main problems are shown as follows:

(1) Traditional VLAN virtual local area network technology can only provide cloud data center with 4096 isolated networks, and such network size can not adapt to the demand of large-scale 
cloud data center [6].

(2) Since the scale of cloud data center virtual machine (VM) is increased rapidly, switch MAC table entries can not meet the need easily, thereby increasing the difficulty of IP address allocation and management.

(3) Virtualization technology is used in the cloud computing network, virtual machine migration can be realized, and the utilization rate of network resources can be improved. Since virtual machine migration should be completed dynamically, therefore cloud computing network address management and network strategy migration may suffer from enormous challenges.

(4) When there are multiple cloud computing data center architectures, large two-layer network construction and other problems due to unified network topology management and data center migration of data center should be fully considered.

In the paper, it is proposed that a two-layer data center network based on Overlay SDN should be newly established on the basis of Overlay virtualization network technology and SDN software defined network in order to solve the above problem of traditional three-layer cloud computing data center network architecture.

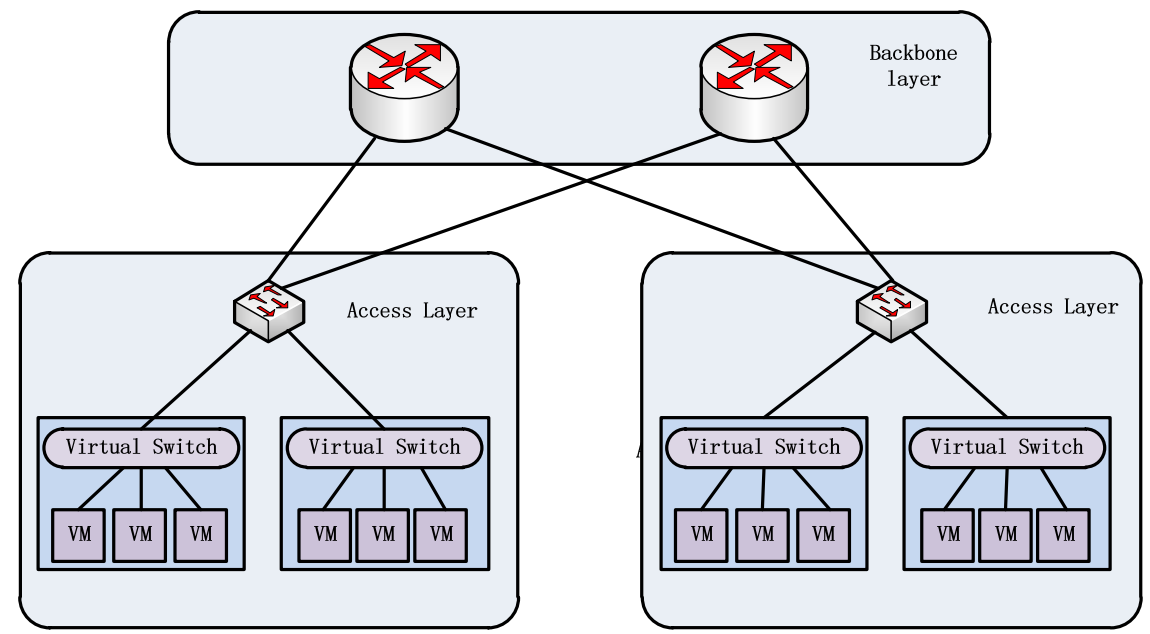

Fig.3. Two-layer cloud computing data center network architecture

A novel cloud computing data center network architecture is constructed through combining SDN technology and Overlay network virtualization technology, therefore function flexibility can be realized through cloud computing network, and communication performance can be further improved.

\section{SDN technology and Overlay virtualization network technology}

SDN technology. Software Defined Network is referred to as SDN. Network equipment control plane is independent from network equipment. In addition, it can be operated on independent central control device [7]. Software pattern is utilized in SDN technology for replacing complicated network control panel based embedded form under traditional pattern. SDN technology has the following core concepts: firstly, separation between network equipment control and forwarding; secondly, network centralized control; thirdly, open API interface and its programming feature. SDN has separation function aiming at network control and data forwarding as shown in Figure 4.

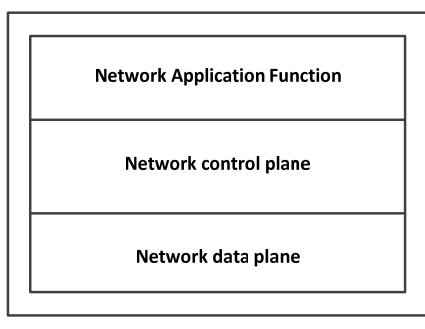

Traditional network equipment

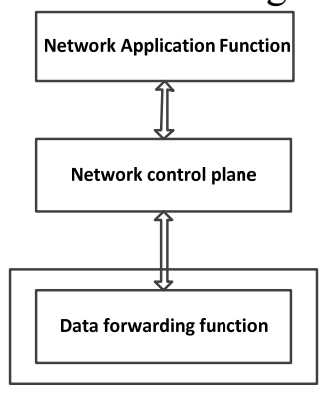

Fig.4. SDN control and forwarding decoupling 
Overlay virtualization network technology. In network technology, Overlay virtualization network technology has the significance of virtualization technology of overlaying one network on the other network. Therefore, foundation network is free from large-scale modification by Overlay virtualization network technology. It has independent network control and forwarding plane. Overlay virtualization network technology is mainly used in large-scale data network interconnection. It is critical technology for realizing cloud computing and network integration [9].

Currently, Overlay virtualization technology mainly includes generic routing encapsulation network virtualization (NVGRE), virtual scalable local area network (VXLAN) and no-state transfer tunnel (STT). The technology comparison is shown in Table 1.

Tab.1. Comparison of three Overlay technologies

\begin{tabular}{|c|c|c|c|c|c|}
\hline Technology & Supporter & $\begin{array}{c}\text { Technology } \\
\text { keys }\end{array}$ & $\begin{array}{c}\text { Network } \\
\text { virtualization mode }\end{array}$ & $\begin{array}{c}\text { Data increase } \\
\text { header length }\end{array}$ & Link Hash ability \\
\hline VXLAN & $\begin{array}{c}\text { Cisco/Vmware, } \\
\text { etc. }\end{array}$ & 1.2 over UDP & $\begin{array}{c}\text { VXLAN header } \\
24 \mathrm{~b} \text { VNI }\end{array}$ & 50 Byte & $\begin{array}{c}1.2 \sim 1.4 \text { HASH can be operated in } \\
\text { current network }\end{array}$ \\
\hline NVGRE & $\begin{array}{c}\text { HP/Microsoft, } \\
\text { etc. }\end{array}$ & 1.2 over GRE & $\begin{array}{c}\text { NVGRE header } \\
\text { 24b VSI }\end{array}$ & 42 Byte & $\begin{array}{c}\text { Network updating is required by } \\
\text { GRE header HASH }\end{array}$ \\
\hline STT & Vmware & 1.2 over TCP & $\begin{array}{c}\text { STT header } \\
\text { 64b Context ID }\end{array}$ & $58 \sim 78$ Byte & $\begin{array}{c}1.2 \sim 1.4 \text { HASH can be operated in } \\
\text { existing network }\end{array}$ \\
\hline
\end{tabular}

In table 1, VXLAN adopts the mechanism of MAC encapsulation in UDP mechanism. Generic routing encapsulation GRE protocol is adopted by NVGRE. Data packet is encapsulated in GRE protocol, which is transmitted through tunnel network. STT belongs to a stateless transport protocol. Network data packets are encapsulated into TCP protocol packet, which are transmitted in tunnel network. The core concept of the three above-mentioned Overlay virtualization network technologies lies in that network data message is transmitted to tunnel level for transmission through some protocol. IP forwarding is set on underlying layer of network, and it is mainly different in different technology selections and different construction tunnel levels. According to comparison in Table 1, it is obvious that equipment should perceive GRE expansion head in NVGRE for implementing HASH on flow ID. Hardware should be updated. STT has larger modification on TCP. The complexity is relatively higher, and VLXAN technology advantage is more obvious. Overlay virtual network structure architecture is shown in figure 5.

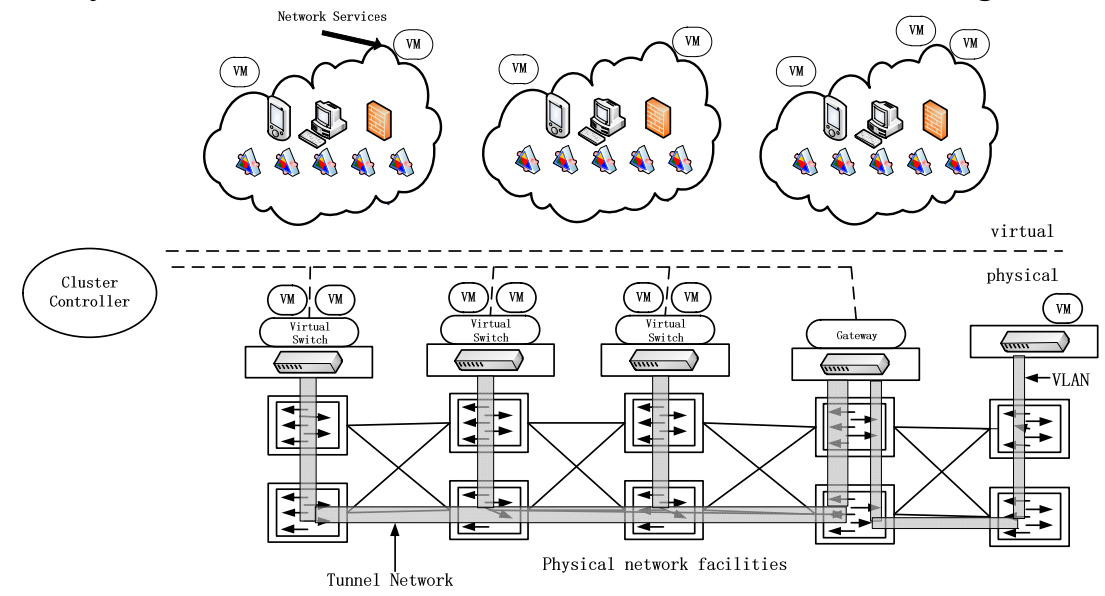

Fig.5. Overlay network virtualization

\section{Realization of cloud computing network architecture based on Overlay SDN}

Data application and physical network coupling are realized by Overlay network virtualization technology. However, network and calculation are two independent parts. Interconnection between network and virtual machine can be realized through current traditional cloud computing network architecture [10]. In the paper, a network architecture implementation plan of cloud computing data center combining SDN technology and Overlay network virtualization technology is proposed. A 
network architecture of network control and management is defined through SDN technology. Therefore, computing resources, storage resources and network resources can be effectively unified. The network architecture also provides an open interface so that the upper application can provide new business through these interfaces.

A cloud computing data center network architecture plan based on Overlay SDN technology in the paper is shown in Figure 6.

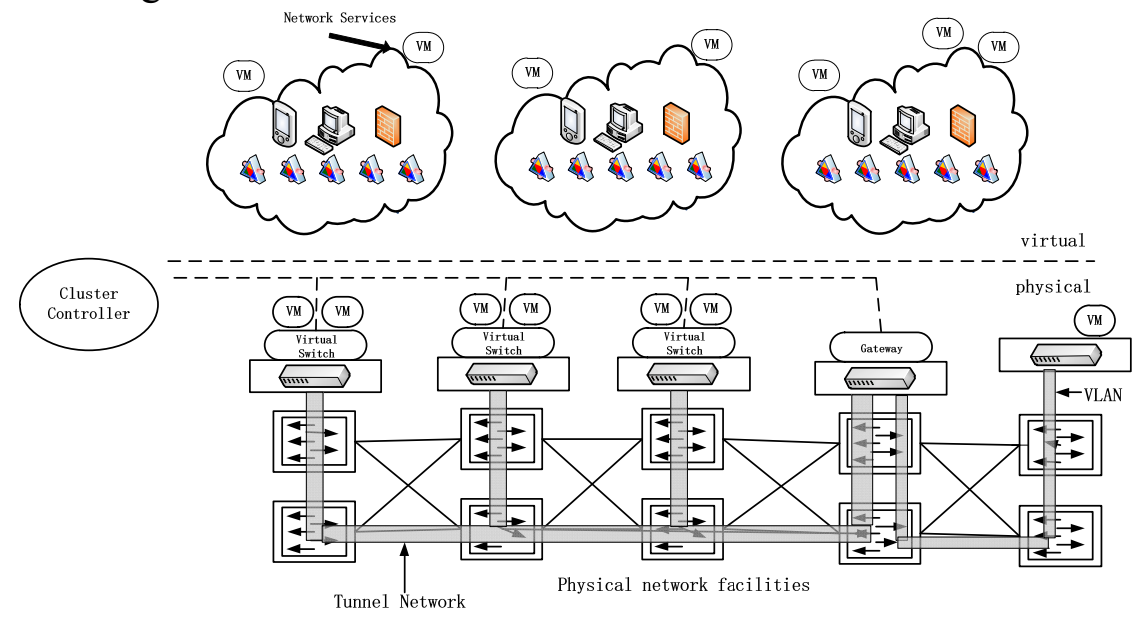

Fig.6. Cloud computing data center network architecture based on Overlay SDN

Overlay network virtualization includes 4 layers, respectively physical network layer, virtual network layer, network control layer and network arranging layer. The physical network layer is jointly composed of access switch, gathering switch, core switch and other equipment. The tenant network can get transparent forwarding paths from the physical network layer; Tenant network layer is composed of virtual networks laminated by different tenants. Network control layer is responsible for completing network information collection, network configuration and flow chart issuance function. Traffic control, network routing information configuration and other service functions are realized on tenant networks. Network service chain is formed in the network through network arrangement layer of Overlay network virtualization. Network arrangement layer also can realize docking with cloud management platform. Network strategy and network service deployment can be automatically adjusted through utilizing network monitoring information, and function of automatically deploying tenant virtual network can be completed.

Overlay SDN technology acts as a mixed network solution. A variety of network technologies are applied, such as network virtualization, network function virtualization (NFV), software definition technology, etc. Firstly, network virtualization technology is utilized for virtual overlay of physical networks, and it is more flexible to create tenant isolated virtual networks. On the basis, software definition technology is added, thereby realizing data forwarding and control separation on virtual network equipment; finally, network function virtualization technology is utilized to realize flexible deployment of network services. In the cloud computing data center network architecture based on Overlay SDN technology proposed in the paper includes the following four key parts.

(1) Virtual switches (vSwitch): it used to realize overlay network technology based on physical network;

(2) Gateway: interconnection and virtual routing between virtual network and physical network, DHCP, NAT and firewall, load balancing and other functions can be realized.

(3) Distributed firewall: it is used for realizing three-layer interconnection distributed router and traffic isolation function. This is the most important function in Overlay SDN technology, which can effectively avoid traffic clamping effect, thereby realizing safe isolation of traffic.

(4) Controller and network manager: It is responsible for virtual network configuration, monitoring, network arrangement, automated deployment and other functions, and efficient management of cloud management platform on resource pool can be realized. 


\section{Conclusion}

Cloud computing is rapidly developed in recent year, computing virtualization, storage virtualization and other network technologies have made more significant progress. Cloud computing data center is essentially a multi-tenant shared computing and storage network resources data center network architecture. Brand-new solution is proposed by SDN technology when it is applied to cloud computing network and developed to cloud computing data center network. In the paper, cloud computing is summarized firstly, and problems and challenges of cloud computing development are proposed. Then, Overlay technology and SDN technology are respectively introduced. Cloud computing data center network architecture based on Overlay SDN technology is proposed. In the paper, Overlay SDN technology is analyzed in details, Overlay technology and SDN technology are combined to provide a new concept and a new method for cloud computing data center solution. Network architecture of cloud computing data center will be further developed and become more mature with development of SDN technology and Overlay technology. However, these network technologies are constantly improved with many defects, and the performance should be further improved. Therefore, architecture of cloud computing data center network still requires deeper exploration and study.

\section{References}

[1] Andreas Voellmy, Junchang Wang. Scalable software defined network controllers. ACM SIGCOMM Computer Communication Review. 2012 (4).

[2] Subharthi Paul, Jianli Pan, Raj Jai. Architectures for the future networks and the next generation Internet: A survey. Computer Communications. 2010 (1).

[3] Li Dan, Chen Guihai, Ren Fengyuan, Jiang Changlin, Xu Mingwei. Research progress and trend of data center network. Journal of Computer, 2014 (02).

[4] Luo Junzhou, Jin Jiahui, Song Aibo, East. Cloud computing-system architecture and key technology. Journal of Communication. 2011 (7).

[5] Xue Cong, Ma Cunqing, Liu Zongbin, etc. A safe SDN controller architecture design. Information Network Security, 2014 (9): 34-38.

[6] Lei Baohua. SDN core technology analysis and practical guide. Beijing: Electronic Industry Press, 2013.

[7] Li L E, Mao Z M, Rexford J. Toward software-defined cellular networks. IEEE EWSDN, 2012:443-465.

[8] KREUTZ D, RAMOS F M V, ESTEVES V P, et al. Software-defined networking: a comprehensive survey. Proceedings of the IEEE, 2015, 103(1): 14-76.

[9] Benson T. CloudNaaS: a cloud networking plaiform for enterprise applieations. Proceedings of ACM Symp Cloud Computing. Caseais, Portugal, 2011.

[10] Open Networking Foundation. Software-Defined Networking: the New Norm for Networks. WhitePaper, 2012. 